\title{
Haploinsufficiency of Parp1 accelerates Brca1- associated centrosome amplification, telomere shortening, genetic instability, apoptosis, and embryonic lethality
}

\author{
X Wang ${ }^{1}$, L Liü ${ }^{2}$ C Montagna ${ }^{3}$, T Ried ${ }^{3}$ and C-X Deng ${ }^{\star, 1}$
}

The breast tumor associated gene-1 (BRCA1) and poly(ADP-ribose) polymerase-1 (PARP1) are both involved in DNA-damage response and DNA-damage repair. Recent investigations have suggested that inhibition of PARP1 represents a promising chemopreventive/therapeutic approach for specifically treating BRCA1- and BRCA2-associated breast cancer. However, studies in mouse models reveal that Parp1-null mutation results in genetic instability and mammary tumor formation, casting significant doubt on the safety of PARP1 inhibition as a therapy for the breast cancer. To study the genetic interactions between Brca1 and Parp1, we interbred mice carrying a heterozygous deletion of full-length Brca1 $\left(B r c a 1^{+/ \Delta 11}\right)$ with Parp1-null mice. We show that Brca1 ${ }^{41 / / 411}$;Parp1 ${ }^{-/-}$embryos die before embryonic (E) day 6.5, whereas Brca1 ${ }^{411 / 411}$ embryos die after E12.5, indicating that absence of Parp1 dramatically accelerates lethality caused by Brca1 deficiency. Surprisingly, haploinsufficiency of Parp1 in Brca $1^{411 / 411}$ embryos induces a severe chromosome aberrations, centrosome amplification, and telomere dysfunction, leading to apoptosis and accelerated embryonic lethality. Notably, telomere shortening in Brca ${ }^{111 / 411 ;}$;Parp $1^{+/}$MEFs was correlated with decreased expression of Ku70, which plays an important role in telomere maintenance. Thus, haploid loss of Parp1 is sufficient to induce lethality of Brca1-deficient cells, suggesting that partial inhibition of PARP1 may represent a practical chemopreventive/therapeutic approach for BRCA1-associated breast cancer.

Cell Death and Differentiation (2007) 14, 924-931. doi:10.1038/sj.cdd.4402105; published online 23 February 2007

Germline mutations of the breast cancer associated gene 1 (BRCA1) cause approximately half of familial breast cancer cases and most of the combined familial breast and ovarian cancers (reviewed in Brody and Biesecker ${ }^{1}$ ). Mounting evidence reveal that BRCA1 plays an essential role in many biological processes, including transcriptional activation and repression, cell cycle regulation, chromatin remodeling, DNAdamage repair, centrosome duplication, and protein transportation (reviewed in $\left\{\right.$ Deng $\left.^{2}, \# 7399\right\}$ ). Consistently, loss of BRCA1/Brca1 in both the human and mouse results in defective DNA-damage repair, abnormal centrosome duplication, impaired homologous recombination $(\mathrm{HR})$, defective cell cycle checkpoints, growth retardation, increased apoptosis, genetic instability, and tumorigenesis (reviewed in Deng $^{2,3}$ ).

Owing to their impaired ability to undergo HR, BRCA1deficient cells cannot effectively repair HR-mediated DNA damage, such as DNA double-strand breaks (DSBs). ${ }^{4,5}$ Defective DNA-damage repair may also make BRCA1deficient cancer cells more sensitive to DNA-damaging agents, such as irradiation, mitomycin $C$ and adriamycin. ${ }^{4,6-8}$ Thus, one of the therapeutic approaches against BRCA1- deficient cancer cells is treating these cells with DNAdamaging-agents to introduce acute DNA damage. Several recent studies indicated that inhibitors for poly(ADP-ribose) polymerase-1 (PARP1) could kill precancerous BRCA1deficient cells with high specificity. ${ }^{9-11}$ PARP1 inhibitors could also efficiently kill several tested cell lines that are derived from human breast cancers or murine mammary tumors. ${ }^{11,12}$

PARP1 is an abundant nuclear protein found in most eukaryotes. ${ }^{13}$ It binds to DNA strand breaks and concomitantly synthesizes oligo- or poly(ADP-ribose) chains that are covalently coupled to various acceptor proteins by transfer of ADP-ribose units from NAD ${ }^{+}$. PARP1 is also involved in DNA-break sensing and signaling when single-strand break (SSB) repair or base excision repair pathways are engaged. ${ }^{14}$ Inhibition of PARP1 activity may result in formation of DSBs when unrepaired SSBs meet replication forks. ${ }^{14}$ Because BRCA1 mutant cells cannot repair DNA DSBs properly, PARP1 inhibition may result in accumulation of DSBs and illegitimated DNA end joining, consequently leading to growth arrest and apoptosis. This may account for the molecular basis why BRCA1-deficient cells are extremely sensitive to PARP1 inhibition.

\footnotetext{
${ }^{1}$ Genetics of Development and Disease Branch, National Institute of Diabetes, Digestive and Kidney Diseases, National Institutes of Health, Bethesda, MD, USA; ${ }^{2}$ Laboratory for Reproductive Medicine, Department of Obstetrics and Gynecology, University of South Florida College of Medicine, Tampa, FL, USA and ${ }^{3}$ Genetics Branch, Center for Cancer Research/NCI/NIH, Bethesda, MD, USA

${ }^{*}$ Corresponding author: C-X Deng, Genetics of Development and Disease Branch, 10/9N105, National Institute of Diabetes, Digestive and Kidney Diseases, National Institutes of Health, Bethesda, MD, USA. Tel: + 301402 7225; Fax: + 301480 1135; E-mail: chuxiad@bdg10.niddk.nih.gov

Keywords: Brca1; Parp1; Ku70; genetic instability; cell death

Abbreviations: BRCA1, Breast cancer associated gene 1; PARP1, Poly(ADP-ribose) polymerase 1; SKY, spectral karyotyping analysis; Q-FISH, quantitative fluorescence in situ hybridization; DSBs, DNA double-strand breaks; SSB, Single-strand breaks; HR, Homologous recombination

Received 18.9.06; revised 11.12.06; accepted 28.12.06; Edited by R De Maria; published online 23.2.07
} 
Murine Parp1 has been disrupted by gene targeting. ${ }^{15}$ Parp1-deficient mice develop normally; however, they suffer spontaneous tumors in multiple organs, including the

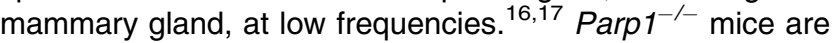
susceptible to chemical carcinogen-induced tumors with increased frequencies. ${ }^{18}$ Parp1 deficiency also induces high levels of aneuploidy, chromosomal fragmentation and fusion as well as chromosome loss and gain. ${ }^{19}$ Consistent with a role of PARP1 in maintaining genetic stability, the absence of p53 or Ku80 significantly enhances tumor formation. ${ }^{16,17}$ These data suggest that PARP1 may serve as a tumor suppressor in mammalian cells and that long-term inhibition of PARP1 may cause, rather than inhibit, breast cancer formation.

In this study, we investigated the genetic interaction between Brca1 and Parp1 through interbreeding Brca1 $1^{411 / \Delta 1120}$ and Parp $1^{-/-15}$ mice generated previously. Our analysis indicates that haploid loss of Parp1 is sufficient to induce apoptosis and inhibit Brca1 mutant cell growth. Our data not only uncover extensive genetic interactions between Brca1 and Parp1 in DNA-damage repair, centrosome duplication, and telomere maintenance, but also suggest that partial inhibition of PARP1 in a combination with other drugs may serve as effective chemopreventive and therapeutic approaches for BRCA1associated cancers.

\section{Results}

Parp1 deficiency accelerates embryonic lethality of Brca1 $^{\text {111/411 }}$ embryos. To study genetic interactions between Brca1 and Parp1, we crossed Brca1 ${ }^{+/ \Delta 11}$ and Parp1 ${ }^{+/}$mice. Previous investigations showed that the majority of $B r c a 1^{411 / \Delta 11}$ embryos died before birth, ${ }^{20}$ whereas Parp $1^{-1-}$ mice survived to adulthood displaying no obvious developmental defects. ${ }^{15}$ Our data indicated that animals double heterozygous for Brca1 and Parp1 mutations were indistinguishable from wild-type controls. However, no Brca1 ${ }^{41 / 411} ;$ Parp $^{-/-}$mice were found among 535 offspring generated from crosses between $B r c a 1^{+/ 411} ;$ Parp $^{+/-}$and Brca1 $^{+/ \Delta 11} ;$ Parp 1 $^{+/-} \quad(n=115)$, and crosses between Brca1 $^{+/ \Delta 11} ;$ Parp1 $^{-/-}$and Brca1 ${ }^{+/ \Delta 11} ;$ Parp1 $^{-/-} \quad(n=420)$ mice (Table 1), suggesting that the $\operatorname{Brca1}^{411 / \Delta 11} ;$ Parp $^{-/-}$ mutation is recessive lethal.
Next, we dissected pregnant females to study the possible phenotypes of $\mathrm{BrCa}^{411 / \Delta 11} ; \mathrm{Parp1}^{-/-}$embryos during gestation. Our analysis of 427 of E6.5-18.5 embryos generated from crosses of the above animals failed to detect any Brca 1 $^{41 / \Delta 11} ;$ Parp 1 $^{-/-}$embryos (Table 1), suggesting that the Brca1 $^{411 / \Delta 11} ;$ Parp $^{-/-}$embryos died before E6.5. Because Brca1 $1^{411 / 411}$ embryos usually did not die before E12.5 (Table 1), these data indicated that the absence of Parp1 accelerated lethality of Brca $1^{411 / \Delta 11}$ embryos.

Next, we studied the Brca1 ${ }^{\Delta 11 / \Delta 11} ; P_{\text {Parp }} 1^{+/-}$embryos and found that they all were significantly smaller than the controls (Figure 1a). Our analysis indicates that although many Brca $1^{411 / 411}$ embryos were smaller than wild-type littermates, their development was relatively normal and they did not die until after E12.5 (Table 1 and not shown). In contrast, we also failed to find any $\mathrm{Brca1}^{111 / \Delta 11} ; \mathrm{Parp}^{+/-}$embryos that were older than E15.5 (Table 1). Moreover, about 33\% (8/24) Brca1 ${ }^{\Delta 11 / \Delta 11} ;$ Parp 1 $^{+/-}$embryos exhibited exencephaly because of failed closure of the anterior neural tube (Figure 1b), whereas about $10 \%$ Brca $1^{\Delta 11 / \Delta 11}$ embryos exhibited a similar defect (not shown). Our previous investigation showed that about $1-2 \%$ of the $B r c a 1^{\Delta 11 / \Delta 11}$ mice survived to adulthood, ${ }^{20-22}$ whereas no Brca1 ${ }^{411 / \Delta 11} ;$ Parp $^{-/-}$and Brca $1^{411 / 411}$; $\mathrm{Parp1}^{+/}$mice were found in 535 offspring generated from our crosses (Table 1). These observations indicate that the absence or haploid loss of Parp1 accelerates embryonic lethality caused by the $B r c a 1^{\Delta 11 / \Delta 11}$ mutation.

Brca1 111/411;Parp1 $^{+/-}$embryos exhibited increased apoptosis. To determine whether altered cell death is responsible for developmental delay and the failure to close neural tubes observed in Brca1 $1^{\Delta 1 / \Delta 11} ; P a r p 1^{+/-}$embryos, we analyzed E9.5. Embryos using terminal transferase dUTP nick end labeling (TUNEL) assay. Our analysis revealed significantly higher rates of apoptosis in the neuroepithelium of the entire neural tube of Brca1 $1^{\Delta 11 / 411} ; P_{\text {Parp }}{ }^{+/-}$embryos compared with that of control embryos (Figure $1 \mathrm{~b}-\mathrm{f}$ ). TUNEL assay of the brains indicated that about $35 \%$ of $\mathrm{Brca}^{411 / \Delta 11}$; Parp $^{+/-}, 12 \%$ of Brca1 ${ }^{411 / 411}, 3 \%$ of Parp1 ${ }^{+/-}$, and $2.5 \%$ of wild-type cells were apoptotic. In contrast, proliferation analysis using bromodeoxyuridine (BrdU) incorporation revealed no significant differences in E9.5 embryos of all different genotypes. These observations suggest that the primary cause of the failure of neural tube closure of the

Table 1 Genotype of animals derived from crosses between Brca1 and Parp1 mutant mice

\begin{tabular}{|c|c|c|c|c|c|c|c|c|c|c|}
\hline $\begin{array}{l}\mathrm{Br}+/ \Delta ; \mathrm{Pa}+/-\mathrm{X} \\
\mathrm{Br}+/ \Delta ; \mathrm{Pa}+/-\end{array}$ & Sub total & $\begin{array}{c}\operatorname{Br} \Delta / \Delta ; \\
\mathrm{Pa}-I-(1 / 16)^{\mathrm{a}}\end{array}$ & $\begin{array}{c}\operatorname{Br} \Delta / \Delta ; \\
\mathrm{Pa}+/-(1 / 8)\end{array}$ & $\begin{array}{c}\mathrm{Br}+/ \Delta ; \\
\mathrm{Pa}-/-(1 / 8)\end{array}$ & $\begin{array}{c}\mathrm{Br}+/ \Delta ; \\
\mathrm{Pa}+/-(1 / 4)\end{array}$ & $\begin{array}{c}\mathrm{Br}+/+; \\
\mathrm{Pa}-/-(1 / 16)\end{array}$ & $\begin{array}{c}\mathrm{Br}+/+; \\
\mathrm{Pa}+/-(1 / 8)\end{array}$ & $\begin{array}{c}\mathrm{Br}+/ \Delta ; \\
\mathrm{Pa}+/+(1 / 8)\end{array}$ & $\begin{array}{c}\mathrm{Br}+/+; \\
\mathrm{Pa}+/+(1 / 16)\end{array}$ & $\begin{array}{c}\operatorname{Br} \Delta / \Delta ; \\
\operatorname{Pa}+/+(1 / 16)\end{array}$ \\
\hline At weaning & 115 & 0 & 0 & 15 & 32 & 7 & 6 & 30 & 25 & 0 \\
\hline E15.5-18.5 & 76 & 0 & 0 & 16 & 22 & 7 & 5 & 17 & 7 & 2 \\
\hline E8.5-14.5 & 198 & 0 & 26 & 25 & 49 & 14 & 26 & 28 & 10 & 20 \\
\hline E6.5-7.5 & 54 & 0 & 8 & 6 & 15 & 4 & 8 & 7 & 4 & 2 \\
\hline $\begin{array}{l}\mathrm{Br}+/ \Delta ; \mathrm{Pa}-/-\mathrm{X} \\
\mathrm{Br}+/ \Delta ; \mathrm{Pa}-/-\end{array}$ & Sub total & \multicolumn{2}{|c|}{$\mathrm{Br} \Delta / \Delta ; \mathrm{Pa}-/-(1 / 4)^{\mathrm{b}}$} & \multicolumn{2}{|c|}{$\mathrm{Br}+/ \Delta ; \mathrm{Pa}-/-(1 / 2)$} & \multicolumn{2}{|c|}{$\mathrm{Br}+/+\mathrm{Pa}-/-(1 / 4)$} & & Resorbed & \\
\hline At weaning & 420 & \multicolumn{2}{|l|}{0} & \multicolumn{2}{|c|}{258} & \multicolumn{2}{|c|}{162} & \\
\hline E10.5-16.5 & 75 & \multicolumn{2}{|l|}{0} & \multicolumn{2}{|c|}{60} & \multicolumn{2}{|c|}{15} & \multirow{2}{*}{\multicolumn{3}{|c|}{$\begin{array}{c}22 \\
9\end{array}$}} \\
\hline E8.5-9.5 & 24 & \multicolumn{2}{|l|}{0} & \multicolumn{2}{|c|}{15} & \multicolumn{2}{|c|}{9} & & & \\
\hline
\end{tabular}

${ }^{a}$ This cross generates a total of nine possible genotypes. Frequency of each genotype is based on the prediction if all embryos can survive. ${ }^{\mathrm{b}}$ This cross generates three possible genotypes at $1: 2: 1$ ratio if all embryos/mice can survive 

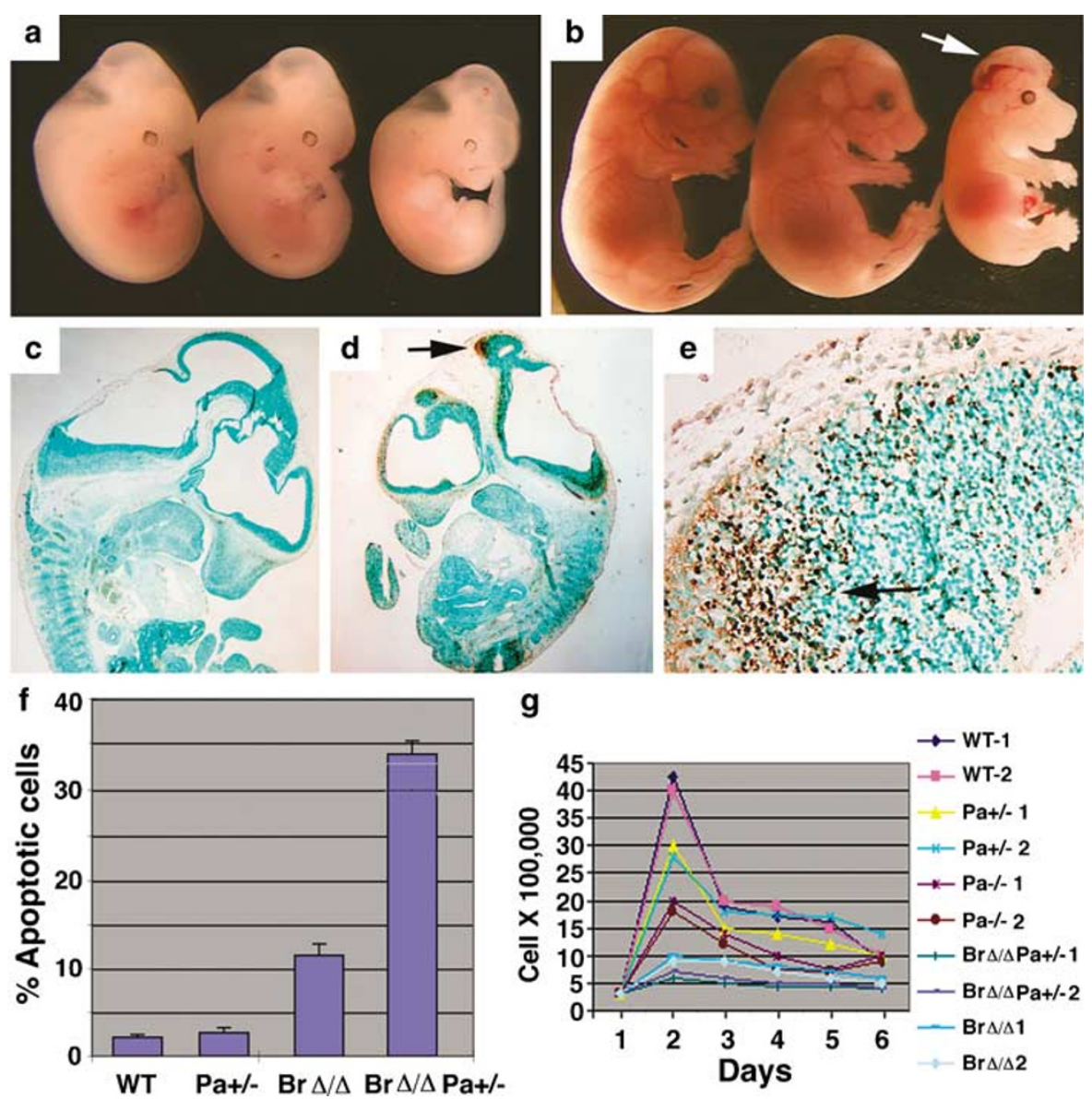

d

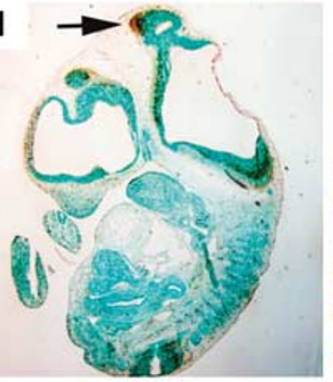

g

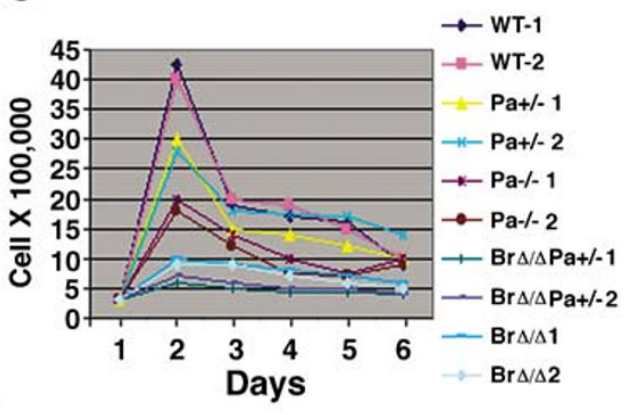

Figure 1 Exencephaly and growth retardation observed in $B r c a 1^{411 / 411} ;$ Parp $1^{+/-}$embryos. (a and b) E9.5 embryos (a), and E14.5 (b) embryos in an order of wild type,

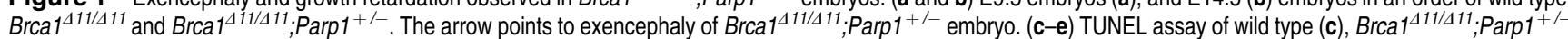
(d) embryos. Midbrain of Brca1 ${ }^{\Delta 11 / 411}$; Parp1 ${ }^{+/-}$embryo (arrow in d) was enlarged (e) to show apoptotic cells (arrow in e). (f) Comparison of apoptotic cells in E9.5 wild-type,

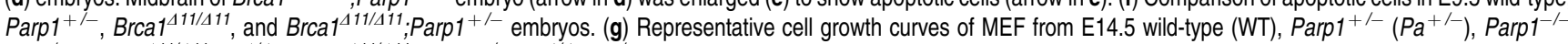

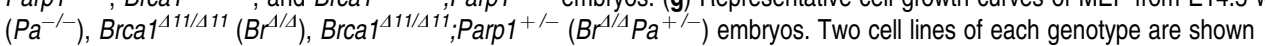

Brca1 $1^{411 / \Delta 11} ; P_{\text {Parp }}{ }^{+/-}$embryos is apoptosis. To study this further, we isolated mouse embryonic fibroblasts (MEFs) from E14.5 embryos with the following genotypes: Brca1 ${ }^{411 / 411}$; Parp 1 $^{+/}$, Parp 1 ${ }^{-/}$, Brca1 $^{411 / 411}$, Parp 1 ${ }^{+/}$, and wild type. We found that $B r c a 1^{\Delta 11 / \Delta 11}$ cells grew poorly; with the removal of one Parp1 allele $\left(\right.$ Brca $^{111 / \Delta 11 ;} ;$ Parp $\left.^{+/-}\right)$, their growth was even poorer (Figure 1g). These results indicate that absence of one allele of Parp1 synergistically increased apoptosis and eventually resulted in the lethality of $B r c a 1^{111 / 411}$ embryos.

\section{Haploid loss of Parp1 and Brca1 deficiency synergistically induce genetic instability. We have shown previously that Brca1 mutant cells exhibited nonclonal accumulations of chromosome aberrations, including chromosome and chromatid breakage. $8,23,24$ To determine whether this chromosome abnormality is enhanced by absence of one allele of Parp1 in Brca1 $1^{411 / 411}$ MEF cells, we studied the chromosomes of Brca1 $1^{11 / 411}$; Parp $1^{+/-}$MEFs by spectral karyotyping analysis (SKY). To minimize the effects from continuous cell culture, we performed SKY on MEFs at passage 1. Our data indicated that nearly all wild-type MEFs (98\%) had a normal karyotype}

(Figure 2a). Chromosome aneuploidy was found in about $5 \%$ of $\operatorname{Parp}^{+/-}$(not shown), 22\% of Brca1 ${ }^{411 / 411}$ (Figure 2b) and $45 \%$ of Brca1411/411;Parp1 ${ }^{+/-}$(Figure 2c) MEFs, respectively. A direct comparison between $B r c a 1^{\Delta 11 / \Delta 11}$ and Brca1 ${ }^{\Delta 11 / 41} ;$ Parp $^{+/-}$cells revealed more severe numerical and structural chromosomal aberrations (Figure $2 \mathrm{~b}-\mathrm{d}$ ). For example, our analysis detected chromosome translocation in $0.03 \%$ Parp1 $^{+/-}, 2.9 \%$ Brca $^{\Delta 11 / 411}$ and $16.9 \%$ Brca $^{411 / 411}$; Parp $^{+/-}$chromosomes (Figure 2d).

Notably, we found that $25 \%$ of Brca $1^{111 / \Delta 11}$ metaphase cells exhibited quadriradial structures in Giemsa-stained chromosome spreads (Figure $2 e$ and $f$ ). With the loss of one allele of wild-type Parp1, this lesion increased to about $60 \%$ $\left(\right.$ Brca1 $^{411 / 411}$;Parp1 ${ }^{+/}$) (Figure 2f). These observations directly demonstrate that Parp1 haploinsufficiency and Brca1 deficiency synergistically accelerate genetic instability through triggering numerical and structural chromosomal abnormalities.

Haploid loss of Parp1 and Brca1 deficiency synergistically induce centrosome amplification. Previous studies revealed that $B r c a 1^{411 / \Delta 11}$ MEFs exhibited centrosome amplification. ${ }^{8,22}$ To determine whether this 


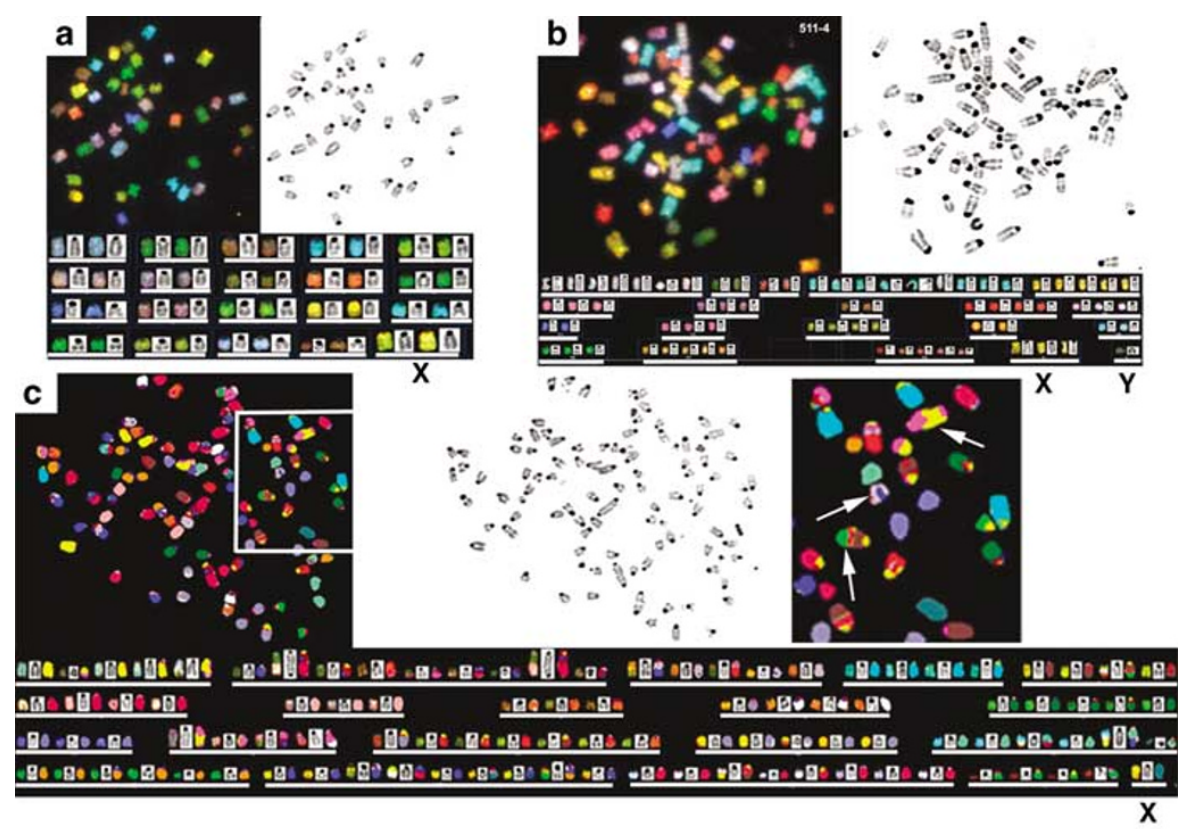

d

\begin{tabular}{l|ccc} 
Genotype & $\begin{array}{l}\text { Total } \\
\text { chromosomes } \\
\text { analyzed }\end{array}$ & $\begin{array}{l}\text { No. chromosome } \\
\text { translocations } \\
\text { and breaks }\end{array}$ & $\begin{array}{l}\text { Translocations } \\
\text { and breas } \\
\text { per chromosome }\end{array}$ \\
\hline $\mathrm{WT}$ & 241 & 0 & 0 \\
$\mathrm{Br}+/+\mathrm{Pa}+/-$ & 299 & 1 & 0.00334448 \\
$\mathrm{Br} \Delta / \Delta \mathrm{Pa}+/+$ & 479 & 14 & 0.02922756 \\
$\mathrm{Br} \Delta / \Delta \mathrm{Pa}+/-$ & 1566 & 264 & 0.16960441
\end{tabular}
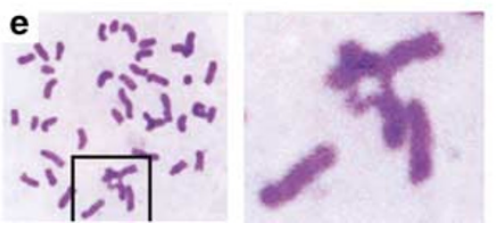

\begin{tabular}{l|c} 
Genotype & Quadriradial \\
\hline $\mathrm{WT}$ & $0 \%$ \\
$\mathrm{Br}+/+\mathrm{Pa}+/-$ & $5 \%$ \\
$\mathrm{Br} \Delta / \Delta \mathrm{Pa}+/+$ & $25 \%$ \\
$\mathrm{Br} \Delta / \Delta \mathrm{Pa}+/-$ & $60 \%$
\end{tabular}

Figure 2 Karyotyping analysis of chromosomes prepared from passage 1 MEFs. (a-c) Metaphases of wild type (a), Brca $1^{\Delta 11 / \Delta 11}$ (b), and Brca ${ }^{\Delta 11 / \Delta 11}$;Parp $1^{+/-}$(c) MEFs. For each panel, the images were shown in displayed pseudocolors of specific spectral ranges (upper left), and black/white (upper right). Chromosomes were also arranged based on the numeric numbers ranging from 1 through $\mathrm{X}$ and $\mathrm{Y}$. MEFs shown in (a) and (c) were derived from female embryos and EMF shown in (b) was derived from a male embryo. Chromosomes belonging to the same group are underlined. The boxed area in (c) is amplified as indicated. Arrows point to chromosome translocation manifested by mixed colors for these chromosomes. (d) A summary showing chromosome breaks and average breaks/chromosome. (e and f) Quadriradial chromosome in

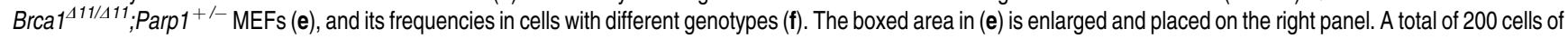
each genotype are counted

abnormality is enhanced by Parp1 haploinsufficiency, we analyzed centrosomes using passage 2 MEF cells of various genotypes. We detected centrosome amplification in Brca1 $^{411 / \Delta 11}$, Parp 1 $^{-/-}$, and Brca1 ${ }^{411 / \Delta 11} ;$ Parp1 $^{+/-}$cells (Figure $3 a-h)$. Quantitative counting indicated that about $40 \%$ of $\mathrm{Brca1}^{411 / \Delta 11}$;Parp1 ${ }^{+/-}$MEF cells contained more than two centrosomes per nucleus, in contrast to about $25 \%$ of $\mathrm{Brca1}^{111 / \Delta 11}$ and $7 \%$ of the Parp ${ }^{+/-}$MEF cells, respectively (Figure $3 \mathrm{i}$ ). This observation indicates that absence of one allele of Parp1 synergistically induces centrosome amplification in Brca1-deficient cells. Our data also revealed centrosome amplification in about $25 \%$ of Parp $^{-1-}$ MEFs. This observation is consistent with a previous finding that absence of Parp1 causes centrosome hyperamplification, ${ }^{25}$ and it provides a basis for the elevated centrosome number in the Brca1 ${ }^{411 / \Delta 11} ;$ Parp $^{+/-}$MEF.
Multiple centrosomes could cause multipolar spindle formation, leading to unequal chromosome segregation, and therefore aneuploidy. Consistent with this, we found about $65 \%$ of Brca1 $^{\Delta 11 / \Delta 11} ;$ Parp1 $^{+/-}$MEFs, $40 \%$ of Brca1 $1^{111 / \Delta 11}$ cells and $10 \%$ of $\mathrm{Parp1}^{+/}$MEFs at passage 2 were aneuploid. In contrast, less than $5 \%$ of aneuploid metaphases were observed in wild-type cells. These observations support the view that faithful segregation of centrosomes is essential in maintaining genome integrity, although other factors may also contribute to genetic stability.

\section{Absence of one allele of Parp1 synergistically affects telomere shortening in Brca1-deficient MEF cells. Telomeres play an important role in stabilizing chromosomes. Previous studies showed that Parp1 is involved in telomere metabolism and Parp1-deficient MEFs}




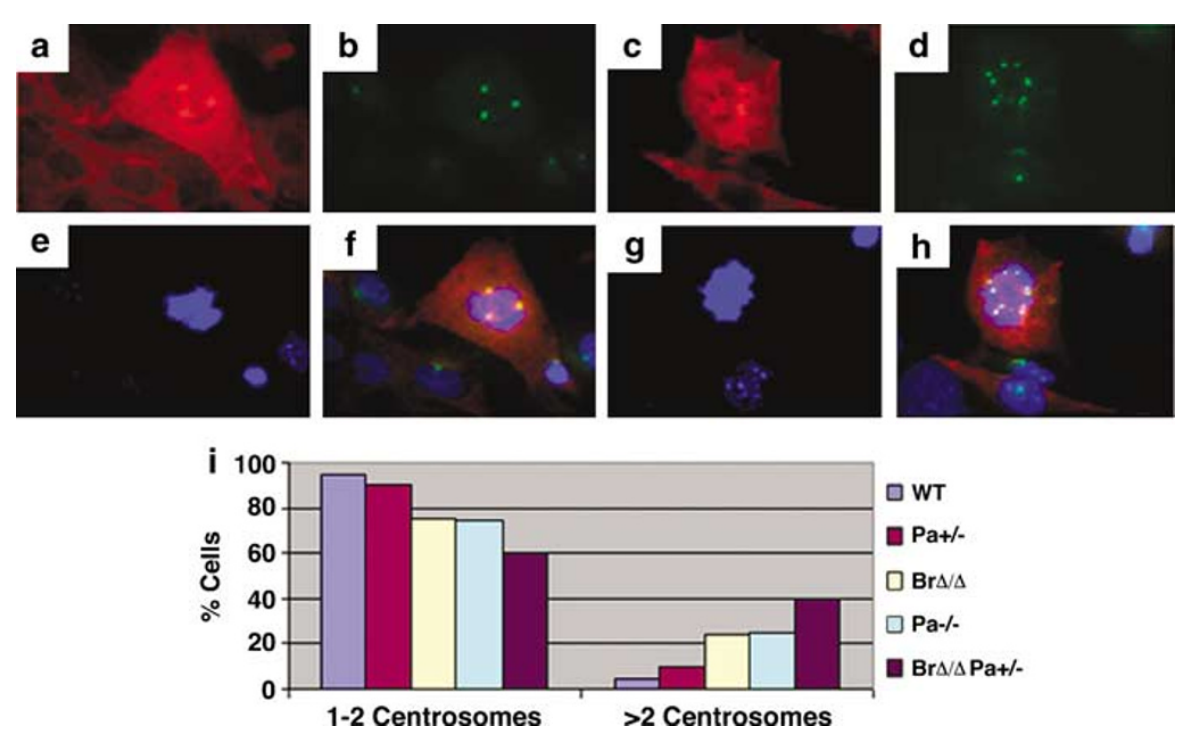

Figure 3 Centrosome amplification in Brca1 and Parp1 mutant MEFs. (a-h) Centrosomes in Brca1 ${ }^{\Delta 11 / \Delta 11}$ (a-d) and Brca $1^{\Delta 11 / \Delta 11 ; P a r p 1^{+/-}}$(e-h) cells revealed by staining with antibodies to $\alpha$-tubulin (a and $\mathbf{e})$, and $\gamma$-tubulin (b and $\mathbf{f}$ ). Images in (c and $\mathbf{g}$ ) are stained with DAPI and the merged images are shown in (d and $\mathbf{h}$ ). (i) Number of centrosome/cell in MEFs at passage 2 of wild-type, Brca $1^{\Delta 11 / \Delta 11}$, Parp ${ }^{+/-}$, Parp $^{-1-}$, and Brca $1^{\Delta 11 / \Delta 11} ;$ Parp $^{+/-}$cells

showed telomere shortening. ${ }^{26}$ T cells derived from Brca1deficient mice display telomere dysfunction characterized by both loss of telomere repeats and defective telomere capping. ${ }^{27}$ This suggests that loss of telomere integrity owing to Brca1 deficiency might contribute to chromosome end dysfunction and permit the formation of potentially oncogenic translocations. By contrast, it has also been reported that disruption of BRCA1 function results in telomere elongation. ${ }^{28}$ On the other hand, our previous study on lymphomas derived from $B \mathrm{Bra}^{411 / 411} ; \mathrm{p5}^{+/ /}$mice detected no changes in telomere length. ${ }^{29}$ However, telomere length in Brca1 mutant MEF cells had not been studied. Therefore, we performed quantitative fluorescence in situ hybridization (Q-FISH) on metaphase chromosomes of primary MEFs of various genotypes derived from breeding of $B r c a 1^{111 /}$

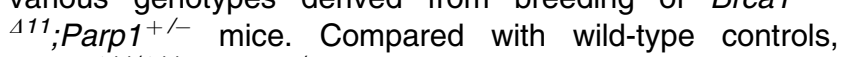
Brca $1^{411 / \Delta 11}$;Parp $1^{+/-}$cells exhibited significantly $(P<0.0001)$ shorter telomeres $(P<0.05)$ (Figure $4 \mathrm{a}, \mathrm{b}$ and d). There were no obvious changes among wild-type, Brca $1^{\Delta 11 / \Delta 11}$ and Parp $1^{+/-}$cells (Figure 4d). Furthermore, we found about $2 \%$ of $B r c a 1^{111 / 411} ;$ Parp $1^{+/-}$cells showing end-to-end fusions (Figure 4b). Three out of $500 \mathrm{Brca} 1^{111 / 411}$; Parp $^{+/}$cells also displayed paired chromosomes (Figure 4c), a rare abnormality that may be caused by uncoupling of chromosome duplication and cell division followed by pairing of homologous chromosomes.

\section{Haploid loss of Parp1 in Brca1 mutant cells decreases} expression of Ku70. We next used a candidate gene approach to check expression of genes that are involved in telomere metabolism. Among a number of candidate genes examined by Western blot analysis, we found that expression of Ku70, which plays an essential role in non-homologous end-joining, telomere maintenance and apoptosis, ${ }^{30}$ was at lower levels in Brca1 $1^{\Delta 11 / 411} ;$ Parp $1^{+/-}$embryos than in embryos with other genotypes (wild-type, Brca $1^{411 / 411}$ or Parp $1^{+/-}$) (Figure 4e). This result suggests that haploid loss of Parp1 in Brca1 ${ }^{411 / 411}$ cells caused downregulation of Ku70. To investigate this further, we performed small interfering RNA (siRNA)-mediated acute suppression of Brca1 in Parp $1^{+-}$cells and compared their Ku70 levels with control cells. Our data indicate that transfection with a Brca1-specific siRNA, but not a control siRNA, results in reduced levels of Ku70 (Figure 4f). We have also performed the same experiment using Brca1 $1^{+/ \Delta 11} ; \mathrm{Parp}^{+/-}$cells and found that Brca1-specific siRNA decreased Ku70 more significantly in these cells (Figure $4 \mathrm{~g}$ ) than in Parp $1^{+/-}$ cells (Figure 4f), perhaps owing to a more efficient knockdown of Brca1 in cells that are already heterozygous for Brca1 mutation. These observations provide strong evidence that Brca1 deficiency and Parp1 haploinsufficiency can cause significant downregulation of Ku70, which is accompanied by telomere shortening, genetic instability, and apoptosis.

\section{Discussion}

We have studied the genetic interactions between Brca1 and Parp1. Our data indicate that Brca1 $1^{11 / 411}$ embryos are extremely sensitive to Parp1 deficiency, as reflected by much earlier embryonic lethality of $B r c a 1^{411 / 411} ;$ Parp $^{-/-}$embryos than Brca1 ${ }^{411 / 411}$ embryos. Brca1 mutant embryos missing only one copy of Parp1 also exhibit severe chromosomal aberrations, centrosome amplification, and telomere dysfunction, which triggers a high incidence of apoptosis and accelerated embryonic lethality. These data indicate that Brca1 and Parp1 work synergistically in maintaining genetic stability. Previous investigations revealed that BRCA1 plays essential functions in multiple types of DNA-damage repair, including DSB repair, non-homologous end joining, and nucleotide excision repair (reviewed in $\mathrm{Xu}^{5}$ ). The absence of 

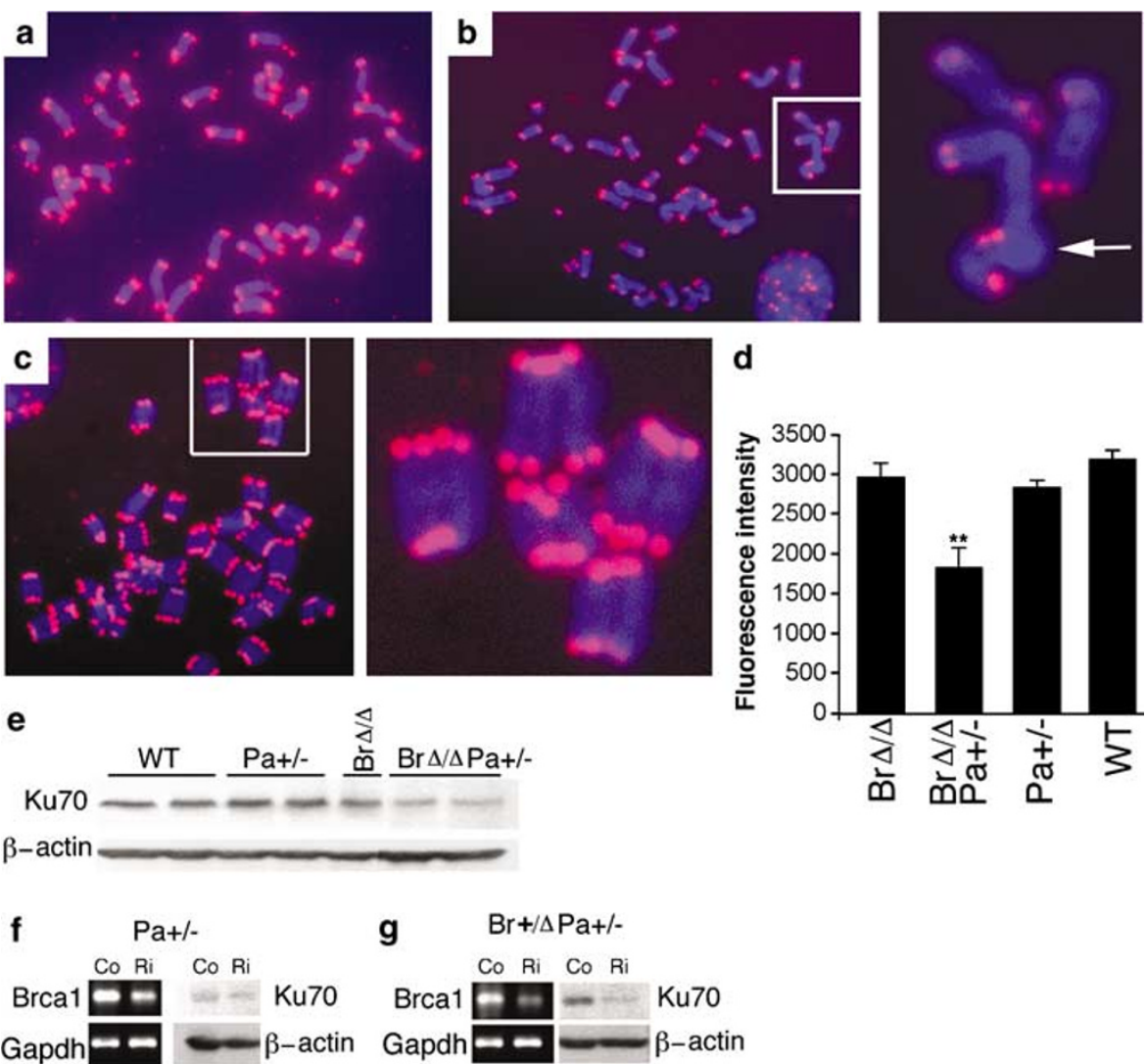

Figure 4 Telomere length and Ku70 expression in MEF cells. (a-c) Telomere-FISH analysis of metaphase chromosomes of wild type (a) and Brca $1^{\Delta 11 / 411 ; P a r p 1^{+/-}}$(b and c) primary MEF cells. Unseparated homological chromosomes are shown in (c). The arrow points to chromosome end-to-end fusion. Boxed areas in (b and c) were enlarged and placed on the right panels, respectively. (d) Quantification of the telomeric FISH signals on individual chromosomes. (e) Western blot analysis of expression levels of Ku70 in embryos of wild-type, Brca1 ${ }^{411 / \Delta 11}$, Parp $1^{+/-}$, and Brca $1^{111 / \Delta 11 ; P a r p 1^{+/-}}$. (f and g) Acute suppression of Brca1 by Brca1 specific siRNA (Ri), but not control siRNA (Co) downregulates Ku70. RNAs and proteins were prepared $48 \mathrm{~h}$ after transfection in Parp $1^{+/-}$(f) and Brca1 ${ }^{+/ \Delta 11} ;$ Parp $^{+/-}$(g) MEFs

BRCA1, therefore, results in accumulation of chromosome damage, cell cycle dysfunction and apoptosis, leading to developmental abnormalities, premature aging, and tumorigenesis. $2,5,21,31$ However, PARP1 is primarily involved in repairing SSBs in DNA. These SSBs, when they meet replication forks, result in the formation of DSBs, which cannot be repaired efficiently in cells that lack functional BRCA1. This may account for the reason why Brca $1^{\Delta 11 / \Delta 11}$ embryos are extremely sensitive to Parp1 deficiency triggering embryonic lethality. In contrast, our finding that Brca1 ${ }^{411 / 411} ;$ Parp 1 $^{+/-}$embryos also exhibited extensive genetic instability, reflecting a dosage-dependent effect of Parp1 in repairing SSBs, although such a reduced ability to repair SSBs in Parp1 ${ }^{+/-}$cells does not cause any apparent problems until Brca1 is also mutated.

Brca1 ${ }^{\Delta 11 / \Delta 11} ;$ Parp $1^{+/}$cells also exhibited marked telomere shortening, which may not be simply attributed to the impaired DNA-damage repair ability in these cells. Instead, these data suggest that Brca1 and Parp1 may play a role in a common pathway maintaining the telomere. Telomeres are tracts of repetitive DNA at each chromosome end and their main function is to prevent chromosome fusion during mitosis thereby stabilizing the genome. Recent studies implicated both BRCA1 and PARP1 in telomere maintenance and function, yet the actual role of BRCA1 remains controversial. $^{27,32,33}$ It has been shown that BRCA1 inhibits the expression of hTERT, the catalytic subunit of telomerase; therefore expression of wild-type BRCA1, but not a tumorassociated mutant BRCA1 (T300G), caused telomere shortening in human prostate and breast cancer cell lines. ${ }^{33}$ In contrast, it was shown in mouse T cells that Brca1 deficiency results in telomere shortening that is characterized by the loss of telomere repeats and defective telomere capping. ${ }^{27} \mathrm{~A}$ recent study indicated that telomere status associated with BRCA1 might vary according to different type of cells. French et $a l^{28}$ indicated that disruption of BRCA1 using a dominantnegative mutant form of BRCA1 (trBRCA1) in telomerasepositive mammary epithelial cells resulted in an increase in telomere length, whereas overexpression the same construct in GM847, a cell line derived from malignant $T$ cells, did not induce any change in telomere length. This report is consistent with our previous finding that loss of Brca1 in Tcell lymphoma did not have an apparent impact on telomere length. ${ }^{29}$ Although it is unclear why BRCA1 deficiency in different types of cells has opposite consequences on telomere length, it is known that BRCA1 binds to a number of factors that may have distinct functions in telomere maintenance. For example, BRCA1 interacts with both 
telomeric repeat binding factor 1 (TRF1) and TRF2, which bind double-stranded telomeric DNA and regulates telomere length. ${ }^{28}$ By expressing mutant versions of these proteins, it was demonstrated that TRF1 is a negative regulator of telomere length, whereas TRF2 plays an essential role in protecting telomeric integrity. ${ }^{34}$ In the MEF cells we studied here, the absence of Brca1 alone does not affect telomere length. However, Brca1 $1^{41 / 411} ;$ Parp $^{+/-}$MEFs displayed telomere shortening and chromosome end fusion that is accompanied by reduced levels of Ku70. Furthermore, Parp1 $^{+/-}$MEF cells which carry an acute suppression of Brca1 also exhibit reduced protein levels of Ku70, suggesting that the reduction of Ku70 is not a secondary consequence owing to the effects of prolonged culture. Thus, our study reveals a synergistic function of Brca1 and Parp1 in maintaining telomeres through modulating expression of Ku70, which forms a complex with Ku80 and localizes to the telomere. ${ }^{30,32}$

Of note, previous investigations revealed physical or genetic interactions between PARP1 and a number of important molecules, including Ku70/80, ${ }^{16}$ DNA-Pkcs, ${ }^{35}$ p53, ${ }^{17}$ and Parp1-Wrn. ${ }^{36}$ Consistent with these interactions, the absence or inhibition of each of these genes in PARP1 mutant mice or mutant cells synergistically enhanced cell death, developmental abnormalities, DNA-damage repair deficiency, genetic instability, and/or tumorigenesis. Our finding revealing the genetic interaction between BRCA1 and PARP1 underscores an important role of BRCA1, PARP1 and their interacting proteins in these processes.

On the basis of the synergistic role of BRCA1 and PARP1 in DNA-damage repair, recent studies suggest PARP1 inhibition as a very promising targeted therapy for BRCA1/2-associated breast cancers. It was demonstrated that PARP-1 inhibitors kill BRCA1/2-deficient cells with extremely high efficiency, whereas the BRCA wild-type cells are relatively nonresponsive to the treatment. ${ }^{9,10}$ Our recent studies have confirmed that PARP1 inhibitors kill Brca1 mutant cells at premalignant stages with high specificity. However, such killing is not obvious in cancer cells because further genetic alteration occurred during tumorigenesis that renders the Brca1-/resistant to Parp1 inhibition. ${ }^{11}$ On the basis of this finding, we proposed that PARP1 inhibitors may be useful for chemoprevention; however, a combination of other therapeutic drugs may be needed for therapeutic treatment of breast cancers. $^{12}$

It is generally believed that PARP1 inhibitors are safe drugs for cancer treatment because Parp1-deficient mice are normal. However, a previous investigation revealed that although PARP1 deficiency does not affect normal development in mice, Parp $1^{-/-} ; p 53^{+/-}$mutant mice suffered high frequencies of several types of tumors, including mammary, prostate, and lung cancers, squamous cell carcinoma, ependymoma, lymphoma, and sarcoma. The mammary glands of both Parp1 $^{-1-} ; p 53^{+/-}$and Parp $^{-1-} ; p 53^{-/-}$mice are normal; however, nine out of $17 \mathrm{Parp}^{-/-} ; p 53^{+/-}$females developed mammary adenocarcinoma by the age of 20 months, whereas $P a r p 1^{-1-} ; p 53^{-/-}$female mice exhibit a high frequency of preneoplastic lesions during early postnatal stages of life. ${ }^{17}$ These observations indicated that a complete and prolonged inhibition of PARP1 might cause severe consequences, including breast cancer development. Our finding that haploinsufficiency of Parp1 sensitizes Brca $1^{411 / 411}$ cells to cellular lethality is very important in this aspect. It suggests that there is no need to completely inhibit Parp1 to achieve cell death. Thus, haploid loss of Parp1 is sufficient to induce lethality of Brca1-deficient cells, suggesting that partial inhibition of PARP1 may represent a practical chemopreventive/therapeutic approach for BRCA1-associated breast cancer.

\section{Materials and methods}

Mice. Maintaining and genotyping of $B r c a 1^{+/ \Delta 11}$ and $P a r p 1^{+/-}$mutant mice were as described. ${ }^{15,20}$ These mice were crossed to generate Brca1 and Parp1 double heterozygous mice, which were further crossed to generate embryos with various genotypes. The background is a mix of 129SVEV, B6C57, and Black Swiss at an approximately $1: 1: 1$ ratio. All experiments were approved by the Animal Care and Use Committee of National Institute of Diabetes, Digestive and Kidney Diseases (ACUC, NIDDK).

Cell cultures and treatments. Primary MEFs were obtained from E14.5 embryos using a standard procedure. The MEFs were cultured in Dulbecco's modified Eagle's medium supplemented with 10\% fetal bovine serum and glutamine. For siRNA transfection, we plated $2.5 \times 10^{5}$ cells onto a 60 -mm dish 1 day before transfection. SiRNA specific for Brca1 (Made by Dharmacon Research, Lafayette, USA) and control siRNA (made by NIDDK) at a concentration of $0.36 \mu \mathrm{M}$ were transfected into MEF cells at passage 2 using oligofectamine as described. ${ }^{22}$ After transfection, cells were harvested at $48 \mathrm{~h}$ and processed for Western blotting and/or reverse transcription (RT)-PCR. The siRNA sequence for murine Brca1 was GAGACAGUAACUAAGCCAG. RT-PCR analysis was carried out using the following primers:

Brca1-1: $5^{\prime}$-CT CAA GAA GCT GGA GAT GAA GG-3'

Brca1-2: 5'-CAA TAA ACT GCT GGT CTC AGG-3'Gapdh-1: 5'-ACA GCC GCA

TCT TCT TGT GC-3'Gapdh-2: 5'-TTT GAT GTT AGA GGG GTC TGC-3'

Apoptosis and proliferation analysis. Sections from E9.5 to E12.5 were analyzed for apoptosis using the ApoTag kit (Intergen Co., Purchase, NY, USA) as recommended by the manufacturer. To evaluate cell proliferation rates, BrdU incorporation was measured using a cell proliferation kit (Amersham Biosciences) following the manufacturer's directions.

MEFs preparation, centrosome staining, and chromosome spread. Primary MEFs were obtained from E14.5 embryos using a standard procedure. Staining for centrosomes in MEFs grown on chamber slides (Falcon, Los Angeles, USA) was performed as described. ${ }^{8}$ Chromosome spread was performed as described. ${ }^{37}$ Briefly, the MEFs were incubated with colcemid $(0.1 \mu \mathrm{g} / \mathrm{ml})$ at $37^{\circ} \mathrm{C}$ for $1 \mathrm{~h}$. Then cells were trypsinized and resuspended gently in $0.56 \% \mathrm{KCl}$, and incubated at $37^{\circ} \mathrm{C}$ for $10 \mathrm{~min}$. The cells were then fixed in a $3: 1$ mixture of methanol acetic acid, dropped across the surface of a slide. Then, cells were spread and allowed to dry on the surface followed by Giemsa (Sigma, Milwaukee, USA) staining.

Western blot analysis. Western blot analysis was accomplished according to standard procedures by ECL detection (Amersham Biosciences, Piscataway, USA). Blots were probed with primary antibody Ku-70 (Santa Cruz Biotechnology, Santa Cruz, USA) followed by $\beta$-actin as a loading control. Peroxidase-labeled goat antirabbit $\lg \mathrm{G}(\mathrm{H}+\mathrm{L})$ and goat anti-mouse $\lg \mathrm{G}(\mathrm{H}+\mathrm{L})$ antibody (Kirkegarrd \& Perry Laboratories, Gaithersburg, USA) were used as secondary antibodies, developed with $\mathrm{ECL}$ detection system.

Q-FISH. Telomere Q-FISH with a telomere probe was performed on chromosome spreads, as described previously. ${ }^{38}$ Telomeres were denatured at $80^{\circ} \mathrm{C}$ for $3 \mathrm{~min}$ and hybridized with Cy3-labeled (CCCTAA) $)_{3}$ peptide nucleic acid probe (DAKO Corporation, Capinteria, USA), washed and mounted in Vectashield mounting medium mixed with $0.5 \mu \mathrm{g} / \mathrm{ml} \mathrm{4}$ 4-6-diamidino-2-phenylindole (DAPI). Telomeres were detected using a Cy3 filter with a Zeiss fluorescence microscope (Carl Zeiss Meditec, Dublin, USA) and images were captured by an AxioCam using AxioVision 3.0 software. Telomere fluorescence intensity was integrated for 
quantitative measurement of relative telomere length using the TFL-TELO program, ${ }^{39}$ kindly provided by $\mathrm{P}$ Lansdorp, and calibrated with fluorescence beads.

SKY analysis. Spectral karyotyping was performed as described.$^{40}$ Briefly, flow sorted normal mouse chromosomes were labeled with specific fluorochromes or fuorochrome combinations. After in situ hybridization images were acquired using an epifuorescence microscope (DMRXA, Leica, Germany) connected to an imaging interferometer (SD200, Applied Spectral Imaging, Israel). Chromosomes were unambiguously identified using a spectral classification algorithm that results in the assignment of a separate classification color to all pixels with identical spectra. Six to 10 metaphases were analyzed for each tumor.

Acknowledgements. We thank members of Deng laboratory for critical reading and discussion of the manuscript. We thank Dr. ZQ Wang for providing Parp1-deficient mice. This work was supported by the intramural Research Program of National Institute of Diabetes, Digestive and Kidney Diseases, National Institutes of Health, USA

1. Brody LC, Biesecker BB. Breast cancer susceptibility genes. BRCA1 and BRCA2. Medicine (Baltimore) 1998; 77: 208-226.

2. Deng CX. BRCA1: cell cycle checkpoint, genetic instability, DNA damage response, and cancer evolution. Nucleic Acids Res 2006; 34: 1416-1426.

3. Deng CX. Tumor formation in Brca1 conditional mutant mice. Environ Mol Mutagen 2002 39: 171-177.

4. Moynahan ME, Cui TY, Jasin M. Homology-directed dna repair, mitomycin-c resistance and chromosome stability is restored with correction of a Brca1 mutation. Cancer Res 2001; 61: 4842-4850.

5. Xu X, Aprelikova O, Moens P, Deng CX, Furth PA. Impaired meiotic DNA-damage repair and lack of crossing-over during spermatogenesis in BRCA1 full-length isoform deficient mice. Development 2003; 130: 2001-2012.

6. Brodie SG, Xu X, Qiao W, Li WM, Cao L, Deng CX. Multiple genetic changes are associated with mammary tumorigenesis in Brca1 conditional knockout mice. Oncogene 2001; 20: 7514-7523.

7. Kim SS, Cao L, Li C, Xu X, Huber LJ, Chodosh LA et al. Uterus hyperplasia and increased carcinogen-induced tumorigenesis in mice carrying a targeted mutation of the Chk2 phosphorylation site in Brca1. Mol Cell Biol 2004; 24: 9498-9507.

8. Xu X, Weaver Z, Linke SP, Li C, Gotay J, Wang XW et al. Centrosome amplification and a defective G2-M cell cycle checkpoint induce genetic instability in BRCA1 exon 11 isoform-deficient cells. Mol Cell 1999; 3: 389-395

9. Bryant HE, Schultz N, Thomas HD, Parker KM, Flower D, Lopez E et al. Specific killing of BRCA2-deficient tumours with inhibitors of poly(ADP-ribose) polymerase. Nature 2005; 434: 913-917.

10. Farmer $\mathrm{H}, \mathrm{McC}$ abe $\mathrm{N}$, Lord CJ, Tutt AN, Johnson DA, Richardson TB et al. Targeting the DNA repair defect in BRCA mutant cells as a therapeutic strategy. Nature 2005; 434: 917-921.

11. De Soto JA, Wang $X$, Tominaga $Y$, Wang $R H$, Cao $L$, Qiao $W$ et al. The Inhibition and Treatment of Breast Cancer with Poly (ADP-ribose) Polymerase (PARP-1) Inhibitors. Int J Biol Sci 2006; 2: 179-185.

12. De Soto JA, Deng C. PARP-1 inhibitors, are they the long-sought genetically specific drugs for BRCA1/2-associated breast cancers? Int J Med Sci 2006; 3: 117-123.

13. Oei SL, Keil C, Ziegler M. Poly(ADP-ribosylation) and genomic stability. Biochem Cell Biol 2005; 83: 263-269.

14. Dantzer F, Schreiber V, Niedergang C, Trucco C, Flatter E, De La Rubia G et al. Involvement of poly(ADP-ribose) polymerase in base excision repair. Biochimie 1999; 81 $69-75$

15. Wang ZQ, Stingl L, Morrison C, Jantsch M, Los M, Schulze-Osthoff $\mathrm{K}$ et al. PARP is important for genomic stability but dispensable in apoptosis. Genes Dev 1997; 11: 2347-2358

16. Tong WM, Cortes U, Hande MP, Ohgaki H, Cavalli LR, Lansdorp PM et al. Synergistic role of Ku80 and poly(ADP-ribose) polymerase in suppressing chromosomal aberrations and liver cancer formation. Cancer Res 2002; 62: 6990-6996.
17. Tong WM, Hande MP, Lansdorp PM, Wang ZQ. DNA strand break-sensing molecule poly(ADP-Ribose) polymerase cooperates with p53 in telomere function, chromosome stability, and tumor suppression. Mol Cell Biol 2001; 21: 4046-4054.

18. Shibata A, Kamada N, Masumura K, Nohmi T, Kobayashi S, Teraoka H et al. Parp-1 deficiency causes an increase of deletion mutations and insertions/rearrangements in vivo after treatment with an alkylating agent. Oncogene 2005; 24: 1328-1337.

19. Tong WM, Cortes U, Wang ZQ. Poly(ADP-ribose) polymerase: a guardian angel protecting the genome and suppressing tumorigenesis. Biochim Biophys Acta 2001; 1552 : 27-37.

20. Xu X, Qiao W, Linke SP, Cao L, Li WM, Furth PA et al. Genetic interactions between tumor suppressors Brca1 and p53 in apoptosis, cell cycle and tumorigenesis. Nat Genet 2001; 28: 266-271.

21. Cao L, Kim S, Xiao C, Wang RH, Coumoul X, Wang X et al. ATM-Chk2-p53 activation prevents tumorigenesis at an expense of organ homeostasis upon Brca1 deficiency. EMBO J 2006; 25: 2167-2177.

22. Wang X, Wang RH, Li W, Xu X, Hollander MC, Fornace Jr AJ et al. Genetic interactions between Brca1 and Gadd45a in centrosome duplication, genetic stability, and neural tube closure. J Biol Chem 2004; 279: 29606-29614.

23. Xu X, Wagner KU, Larson D, Weaver Z, Li C, Ried T et al. Conditional mutation of Brca1 in mammary epithelial cells results in blunted ductal morphogenesis and tumour formation [see comments]. Nat Genet 1999; 22: 37-43.

24. Weaver Z, Montagna C, Xu X, Howard T, Gadina M, Brodie SG et al. Mammary tumors in mice conditionally mutant for Brca1 exhibit gross genomic instability and centrosome amplification yet display a recurring distribution of genomic imbalances that is similar to human breast cancer. Oncogene 2002; 21: 5097-5107.

25. Kanai M, Tong WM, Sugihara E, Wang ZQ, Fukasawa K, Miwa M. Involvement of poly(ADP-Ribose) polymerase 1 and poly(ADP-Ribosyl)ation in regulation of centrosome function. Mol Cell Biol 2003; 23: 2451-2462.

26. d'Adda di Fagagna F, Hande MP, Tong WM, Lansdorp PM, Wang ZQ, Jackson SP. Functions of poly(ADP-ribose) polymerase in controlling telomere length and chromosomal stability. Nat Genet 1999; 23: 76-80.

27. McPherson JP, Hande MP, Poonepalli A, Lemmers B, Zablocki E, Migon E et al. A role for Brca1 in chromosome end maintenance. Hum Mol Genet 2006; 15: 831-838.

28. French JD, Dunn J, Smart CE, Manning N, Brown MA. Disruption of BRCA1 function results in telomere lengthening and increased anaphase bridge formation in immortalized cell lines. Genes Chromosomes Cancer 2006; 45: 277-289.

29. Bachelier R, Xu X, Wang X, Li W, Naramura M, Gu H et al. Normal lymphocyte development and thymic lymphoma formation in Brca1 exon 11-deficient mice. Oncogene 2003; 22: 528-537.

30. Pastwa E, Blasiak J. Non-homologous DNA end joining. Acta Biochim Pol 2003; 50: 891-908.

31. Wang $\mathrm{RH}, \mathrm{Yu} \mathrm{H}$, Deng $\mathrm{CX}$. A requirement for breast-cancer-associated gene 1 (BRCA1) in the spindle checkpoint. Proc Natl Acad Sci USA 2004; 101: 17108-17113.

32. Hande MP. DNA repair factors and telomere-chromosome integrity in mammalian cells. Cytogenet Genome Res 2004; 104: 116-122.

33. Xiong J, Fan S, Meng Q, Schramm L, Wang C, Bouzahza B et al. BRCA1 inhibition of telomerase activity in cultured cells. Mol Cell Biol 2003; 23: 8668-8690.

34. van Steensel B, Smogorzewska A, de Lange T. TRF2 protects human telomeres from end-to-end fusions. Cell 1998; 92: 401-413.

35. Bryant HE, Helleday T. Inhibition of poly (ADP-ribose) polymerase activates ATM which is required for subsequent homologous recombination repair. Nucleic Acids Res 2006; 34: $1685-1691$.

36. Deschenes F, Massip L, Garand C, Lebel M. In vivo misregulation of genes involved in apoptosis, development and oxidative stress in mice lacking both functional Werner syndrome protein and poly(ADP-ribose) polymerase-1. Hum Mol Genet 2005; 14: 3293-3308

37. Deng $\mathrm{CX}, \mathrm{Xu} \mathrm{X}$. Generation and analysis of Brca1 conditional knockout mice. Methods $\mathrm{Mol}$ Biol 2004; 280: 185-200.

38. Hande MP, Samper E, Lansdorp P, Blasco MA. Telomere length dynamics and chromosomal instability in cells derived from telomerase null mice. J Cell Biol 1999; 144: 589-601.

39. Poon SS, Martens UM, Ward RK, Lansdorp PM. Telomere length measurements using digital fluorescence microscopy. Cytometry 1999; 36: 267-278

40. Liyanage M, Coleman A, du Manoir S, Veldman T, McCormack S, Dickson RB et al. Multicolour spectral karyotyping of mouse chromosomes. Nat Genet 1996; 14: 312-315. 